\title{
Nella naRrativa brasiliana ATtUALE, PROTAGONISTA LA CITTÀ.
}

\begin{abstract}
Amina Di Munno*
ABSTRACT: Il proposito del presente articolo è quello di mettere in luce la presenza e il ruolo della città, non tanto come spazio urbano contrapposto a quello rurale-bucolico, ma di una città che assume una posizione privilegiata, da protagonista o, quanto meno, da coprotagonista, in romanzi prodotti tra la fine del secolo scorso e l'attualità nel panorama letterario brasiliano. Storia, finzione, mito, memoria sono aspetti già presenti nella città descritta durante il Modernismo da autori come Carlos Drummond de Andrade, Manuel Bandeira e Vinicius de Moraes, ma anche, in tempi diversi, da Lima Barreto, João do Rio, Rubem Fonseca, testimoni delle trasformazioni di Rio de Janeiro. Dal canto suo Mário de Andrade con l'opera Paulicéia Desvairada rivela, conciliando estetiche differenti, i cambiamenti di São Paulo dovuti anche alle forti immigrazioni.

Tuttavia, l'obiettivo principale di questo testo verte sull'importanza attribuita alle città di Manaus e di Brasilia rispettivamente nei romanzi di Milton Hatoum e João Almino. Questi autori non si inseriscono nel filone del regionalismo, così marcato nella tradizione brasiliana, ma, piuttosto, fanno agire le presenze umane in un contesto cittadino che assume vita propria, quasi personificandosi.
\end{abstract}

PAROLE CHIAVE: città; protagonista; storia; finzione; mito; trasformazioni.

RESUMO: O propósito do presente artigo é desvelar a presença e o papel da cidade, não tanto como espaço urbano contraposto ao rural-bucólico, mas de uma cidade que assume uma posição privilegiada, de protagonista ou, ao menos, de

* Università degli Studi di Genova, Gênova (Itália) -adimunno@gmail.com

Processo n.2013/20971-0 Fundação de Amparo à Pesquisa do Estado de São Paulo (FAPESP)

DOI: http://dx.doi.org/10.11606/issn.2238-8281.v0i34p23-30 
coprotagonista, em romances escritos entre o fim do século passado e a atualidade no panorama literário brasileiro. História, ficção, mito, memória são aspectos já presentes na cidade descrita durante o Modernismo por autores como Carlos Drummond de Andrade, Manuel Bandeira e Vinicius de Moraes, mas também, em tempos diversos, por Lima Barreto, João do Rio, Rubem Fonseca, testemunhas das transformações do Rio de Janeiro. Por seu lado, Mário de Andrade com a obra Paulicéia Desvairada revela, conciliando estéticas diferentes, as mudanças de São Paulo devidas também às grandes imigrações.

Entretanto, o objetivo principal deste texto se debruça sobre a importância atribuída às cidades de Manaus e de Brasilia respectivamente nos romances de Milton Hatoum e João Almino. Estes autores não se inserem no filão do regionalismo, tão marcado na tradição brasileira, mas, muito mais, fazem agir a presença humana num contexto urbano que assume vida própria, quase personificando-se.

PALAVRAS-CHAVE: cidade; protagonista; história; ficção; mito; transformações.

ABSTRACT: The aim of this article is to observe the presence and the role of the city not only as an urban space opposed to the rural one, but of a city that takes on a privileged position, as a protagonist or, at least, as a co-star, in novels produced between the end of last century and our days in the brasilian literary landscape. History, fiction, myth, memory are aspects already present in the city described during the Modernism by authors as Carlos Drummond de Andrade, Manuel Bandeira and Vinicius de Moraes, but also, in different moments, by Lima Barreto, João do Rio, Rubem Fonseca, witnesses of the changes of Rio de Janeiro. On the other hand Mário de Andrade, with his work Paulicéia Desvairada, reveals the changes of São Paulo due also to the great immigration.

Nevertheless the principal purpose of this text is about the importance given to the cities of Manaus and Brasília respectively in Milton Hatoum's and João Almino's novels. These authors do not include themselves in the line of research of Regionalism, typical of the brasilian tradition, but they make the human presences to act in an urban context that seems itself personified.

KEYWORDS: city; protagonist; history; fiction, myth; changes. 
a storia del romanzo e la Storia tout court si intrecciano nel loro cammino. A partire dal Romanticismo, momento in cui hanno inizio le trasformazioni dovute all'industrializzazione, nascono diverse tipologie di romanzi: quello avventuroso, storico, di costume, psicologico, autobiografico. Tuttavia è il romanzo storico a riscuotere maggiore fortuna nei primi decenni dell'Ottocento. Nella narrativa di quegli anni, benché intervengano personaggi reali e di fantasia, protagonista del romanzo è la storia.

Durante la seconda decade del XIX secolo, sorgono i primi romanzi in Brasile, il cui obiettivo principale era quello di creare l'atmosfera e le basi per la costruzione di una cultura autoctona brasiliana. Tali ideali portano via via alla realizzazione del romanzo indianista e storico, a quello regionalista e infine al romanzo urbano. E più di qualunque altro genere letterario narrativo, quest'ultimo raggiunse un grado di notevole successo, grazie soprattutto alla penna di Machado de Assis. Le principali caratteristiche erano quelle volte a fare un ritratto della società coeva, in modo precipuo, della società di Rio de Janeiro, nel cui scenario erano trattati episodi legati a situazioni economiche, sociali e sentimentali non solo e non più esclusivamente delle classi aristocratiche o borghesi della società, ma comuni alla maggior parte delle persone. Nelle opere di questo periodo, la città ha una funzione importante come spazio geografico. In essa avvengono trasformazioni che ne accelerano il processo di urbanizzazione e, tuttavia, non riveste ancora un ruolo da protagonista. Personaggi e protagonisti permangono le figure maschili e femminili 
che la percorrono e ne colmano gli spazi, anzi lo spazio urbano diventa il locus per esprimere la condizione umana.

Storia, finzione letteraria, mito, memoria sono aspetti ben presenti nella città descritta durante il Modernismo in Brasile da autori come Carlos Drummond de Andrade, Manuel Bandeira e Vinicius de Moraes, ma anche, in tempi diversi, da Lima Barreto, João do Rio e più tardi da Rubem Fonseca, testimoni tutti dei mutamenti di Rio de Janeiro. A questo proposito, significativo è il racconto di Rubem Fonseca, A Arte de Andar nas Ruas do Rio de Janeiro, pubblicato nel 2001 in Italia da Robin (collana Biblioteca del vascello) con il titolo L'arte di andare a piedi per le strade di Rio de Janeiro e altri racconti, in cui le strade della città, punto focale, sono il pretesto per mostrare, attraverso la loro frequentazione da parte del personaggio Augusto, varie tematiche legate a situazioni di violenza, di degrado ambientale nonché alle riflessioni circa il ruolo degli abitanti che occupano tali spazi urbani. In definitiva, tramite la società moderna, è la città a parlare di se stessa. Il deambulare, il viaggio, l'idea di città personificata rimandano a un libro noto in Brasile, tradotto in portoghese da Diogo Mainardi, che è Le città invisibili di Italo Calvino. Le 55 città sono immaginarie e hanno nomi di donna. Lo stesso Calvino rilascia informazioni precise su quest'opera di assoluta originalità in una breve autointervista: La città come luogo della memoria e dei desideri.

È un libro che mi sono portato dietro per anni, scrivendo saltuariamente, a intervalli. Sono passato attraverso vari momenti: ora scrivevo solo città contente, ora solo città tristi; c'è stato un periodo in cui paragonavo le città al cielo stellato, allo zodiaco, e in un altro periodo, invece, mi veniva sempre da parlare di spazzatura e immondezzai. Ma non erano solo i cambiamenti d'umore a influire: anche le letture, le discussioni, i quadri, le impressioni visive. (CALVINO, 2002, pp.175-176).

Le città sono allegorie del desiderio, della memoria, della morte, del reale e del trascendente:

Non c'è città piú di Eusapia propensa a godere la vita e a sfuggire gli affanni. E perché il salto dalla vita alla morte sia meno brusco, gli abitanti hanno costruito una copia identica della loro città sottoterra. I cadaveri, seccati in modo che ne resti lo scheletro rivestito di pelle gialla,vengono portati là sotto a continuare le occupazioni di prima. (CALVINO, 1972, p. 53). 
In altre aree cittadine del Brasile, Mário de Andrade con l'opera Paulicéia Desvairada rivela, conciliando estetiche differenti, i cambiamenti di San Paolo dovuti soprattutto alle forti ondate di immigrazione. Carlos Drummond de Andrade conserverà in buona parte della sua opera la memoria della città natale, Itabira, mentre Manuel Bandeira, nel suo libro Libertinagem, specificamente nella poesia "Vou-me embora pra Pasárgada", città dell'antica Persia oggi sito archeologico in Iran, evoca metaforicamente un luogo ideale di felicità dove ogni sogno può essere realizzato. In un quadro referenziale a noi cronologicamente più vicino possiamo delineare nelle opere di Milton Hatoum e di João Almino una mappa narrativa delle città, rispettivamente di Manaus e di Brasilia, all'interno di uno scenario, spazio e tempo che le vede come protagoniste. A partire dai titoli di alcune opere, appare evidente in entrambi gli autori l'importanza che riveste il termine stesso di città: A Cidade Ilhada di Milton Hatoum e Cidade Livre di João Almino.

Le città a cui si fa allusione, contrariamente alle città immaginarie di Calvino, sono città reali, nel primo caso Manaus e nel secondo Brasilia ed esse sono personaggi di primo piano in ogni opera dei due scrittori. Milton Hatoum e João Almino sono narratori conosciuti in Italia, sono stati entrambi tradotti in italiano e i loro romanzi hanno riscosso successo di pubblico e di critica. Moltissime le recensioni soprattutto del romanzo di Hatoum Dois Irmãos, di cui recentemente è stata pubblicata in Italia anche una versione a fumetti, realizzata dai fratelli Fábio Moon e Gabriel Bá. I libri di Milton Hatoum racchiudono nella città di Manaus tradizioni europee, mediterranee e, in perfetta armonia, atmosfere esotiche, tropicali, riti e religioni diverse. Nel romanzo Dois Irmãos di Manaus si nominano strade, piazze, ristoranti, bazar, mercati, centro e periferie, fiumi, palafitte, imbarcazioni. L'epopea familiare è marcata dal conflitto tragico e incessante tra i due gemelli e, tuttavia, come sottolinea Antonio Prete:

Quando il lettore è preso dal gioco intimo e perverso dei due fratelli s'accorge che c'è un altro personaggio che riempie la scena, ed è la città di Manaus - la città stessa di Milton Hatoum- con il molo Escadaria, la praça de Saudade, la rua dos Barés, il mercato, i venditori ambulanti, e soprattutto gli odori forti e i sapori della terra amazzonica affiancati o mescolati agli odori e ai sapori di cibi e bevande che vengono dalla tradizione libanese. (PRETE, A. "Antropologia del doppio"). http://www.zibaldoni.it/2006/11/07/doppio/ acesso: 06/05/2016). 
La città pulsa, è viva, cresce, è soggetta a cambiamenti:

O Café Mocambo fechara, a praça das Acácias estava virando um bazar (...). "Manaus está cheia de estrangeiros, mama. Indianos, coreanos, chineses... $O$ centro virou um formigueiro de gente do interior... Tudo está mudando em Manaus". (HATOUM, 2000, p. 223). (Il Café Mocambo era stato chiuso, praça das Acácias stava diventando un bazar. (...) "Manaus è piena di stranieri, mamma. Indiani, coreani, cinesi... Il centro è diventato un formicaio di gente dell'interno... Tutto sta cambiando a Manaus".

Manaus è luogo simbolico, ma, con i suoi fiumi, i suoi toponimi, i suoni della foresta, è anche l'importante palco, ricco di dettagli identificativi, nel quale agiscono i personaggi.

In Cinzas do Norte, titolo emblematico, metafora di un mondo che finisce in cenere, Manaus è il luogo dell'azione, teatro questa volta dei conflitti fra due famiglie. È una Manaus più politicizzata e ulteriormente aggredita dalla devastazione:

Subimos pelas ruas dos Educandos; na avenida Beira-Rio vimos, lá embaixo, o vazio perto do porto da Escadaria, antes ocupado por um aglomerado de palafitas.

"Sabes onde eles estão?”, perguntou Mundo.

"Eles quem?"

"Os moradores da beira rio. Foram jogados no outro lado da cidade. A área foi toda desmatada, construiram umas casas... Sobrou uma seringueira. Quer dizer, o tronco e uns galhos... a carcaça”. (HATOUM, 2005, pp. 143-144). (Salimmo la rua dos Educandos; nell'avenida Beira-Rio vedemmo, lì giù, il vuoto vicino al porto da Escadaria, prima occupato da un agglomerato di palafitte. "Sai loro dove sono"?, domandò Mundo. "Loro chi”? "Gli abitanti delle sponde del fiume. Sono stati buttati dall'altra parte della città. L'area è stata tutta disboscata, hanno costruito alcune case... È rimasto un albero di caucciù. Cioè, il tronco e qualche ramo... la carcassa"). 
Come la Manaus di Milton Hatoum, anche la Brasilia di João Almino passa per una sorta di processo di "personalizzazione" e assume il ruolo di protagonista nei tre libri che fanno parte di quella che è stata definita la "Trilogia di Brasilia", costituita dai romanzi: Idéias para Onde Passar o Fim do Mundo, Samba-Enredo e As Cinco Estações do Amor, quest'ultimo tradotto in italiano con il titolo Le cinque stagioni dell'amore.

Brasilia è una città ancora senza storia, la sua inaugurazione è avvenuta il 21 aprile 1960 e perciò è tuttora proiettata più verso il futuro che il passato. Essa nasce da una pianificazione strategica, da un progetto di ordine politico. È la Capitale Federale, sede del Governo e, con la sua innovativa architettura, è espressione di modernità, rappresenta la sintesi delle maggiori etnie del paese. Ecco che, come Manaus, essa si presta a ricoprire un ruolo di primaria importanza all'interno di una trama romanzesca. João Almino, tuttavia, non ne mostra solo l'aspetto mitico e grandioso, ma osserva quelli che sono i problemi tipici dei grandi centri urbani come le disuguaglianze sociali, la povertà, la violenza di cui sono testimoni e attori i personaggi fittizi che occupano il centro della scena. Nel romanzo As Cinco Estações do Amor la narrazione è condotta da una voce femminile che mette in luce, tramite il racconto personale e le proprie frustrazioni, le trasformazioni avvenute nel paese negli ultimi trent'anni, passando anche attraverso la dittatura militare:

As cidades adquirem o ar dos tempos por que passam. Brasília, que tinha sido promessa de socialismo e, para mim pessoalmente, de liberdade, não usava mais disfarce. A desolação de suas cidades-satélites já a asfixiava. Respirávamos vinte e quatro horas por dia o ar envenenado da ditadura militar. (ALMINO, 2001, pp. 2122). ( Le città assumono l'aria dei tempi attraverso cui passano. Brasilia, che era stata una promessa del socialismo e, per me personalmente, di libertà, non si travestiva più. La desolazione delle sue città-satellite ormai la asfissiava. Respiravamo ventiquattr'ore su ventiquattro l'aria avvelenata della dittatura militare).

Questa chiave di lettura rimanda a un concetto, ormai diffusosi e studiato nel mondo contemporaneo, che racchiude in una definizione l'idea di "umanizzazione della città". 


\section{Riferimenti bibliografici}

CALVINO, I. Sono nato in America... Interviste 1951-1985, a cura di Luca Baranelli. Introduzione di Mario Barenghi. Milano: Mondadori, 2002.

CALVINO, I. Le città invisibili. Torino: Einaudi, 1972.

PRETE, A. "Antropologia del doppio".

http://www.zibaldoni.it/2006/11/07/doppio/ acesso 06/05/2016.

HATOUM, M. Dois irmãos. Trad. A. Di Munno. São Paulo: Companhia das Letras, 2000.

HATOUM, M. Cinzas do Norte. Trad. A. Di Munno. São Paulo: Companhia das Letras, 2005.

ALMINO, J. As Cinco Estações do Amor. Trad. A. Di Munno. Rio de Janeiro - São Paulo: Editora Record, 2001.

Recebido em 14/01/2017

Aprovado em 14/06/2017 